\section{Most Downloaded Articles}

Mit der Aufnahme in die Literaturdatenbank GeroLit ${ }^{\circledR}$ ist eine weitere Listung von HeilberufeSCIENCE erreicht. Damit steigt auch unsere Verantwortung gegenüber Ihnen als Leser. Dabei stellt sich uns immer wieder die Frage, welche Beiträge oder Themen Sie besonders interessieren. Einen Hinweis auf die Antwort bietet die Rubrik „Most Downloaded Articles" auf der Website von HeilberufeSCIENCE (www.springer.com/medicine/ journal/16024). Hier finden sich - frei zugänglich - die Download-Rates einzelner Artikel für die zurückliegenden 7, 30 oder 90 Tage. Ein Schwerpunkt ist erkennbar: Die Pflegemanagement-Themen finden gegenwärtig Ihr stärkstes Interesse.

Aber auch die Abstracts der Vorträge und Poster des 8. Gesundheitspflegekongresses im November 2010 in Hamburg sind regelmäßig dabei. Daher möchte ich die Gelegenheit nutzen und Sie auf das "Call for Abstracts" für das 3. HeilberufeSCIENCE-Symposium auf dem 9. Gesundheitspflege-Kongress in Hamburg (25.-26. November 2011) hinweisen. Die Abstracts der Poster und Vorträge werden auch dieses Jahr wieder zitierfähig in einer HeilberufeSCIENCE-Sonderausgabe veröffentlicht. Wenn Sie sich inhaltlich beteiligen möchten, würde es mich freuen, wenn Sie Ihr Abstract bis zum 31. August 2011 einreichen. Weitere Informationen finden Sie auf der Website der Heilberufe-Kongresse unter www. heilberufe-kongresse.de.

Die neue Ausgabe von HeilberufeSCIENCE startet mit einem Artikel von Schadenhofer und Stummer über die Untersuchungsergebnisse einer Querschnittsstudie zur genderspezifischen Vulnerabilität für Burn- out in der Gesundheits- und Krankenpflege. Daran schließt sich der Beitrag von Drescher mit den Ergebnissen einer Analyse zur seniorengerechten Gestaltung von Bildschirmeingabemasken beim OnlineBanking und bei Geldausgabeautomaten an, ein aufgrund des demografischen Wandels zunehmend wichtiger werdendes Thema. Auch die Studie von Stellwag und Seeberger widmet sich den Auswirkungen des demografischen Wandels: die Bedeutung des Sports für ältere aktive Golfspieler. Und die Übersichtsarbeit von Meiners et al. befasst sich mit dem derzeitigen Forschungsstand zur Rolle von älteren Menschen als Konsumenten. Abschließend haben wir wieder eine wissenschaftliche Kurzmitteilung, diesmal von Katharina von Lindeman et al. mit den Ergebnissen einer Querschnittsstudie zu Ernährungsgewohnheiten und Diätversuchen von Auszubildenden in Gesundheitsfachberufen, vorrangig in der Krankenpflege.

An dieser Stelle darfich Sie nun wieder einladen, die zweite Ausgabe von HeilberufeSCIENCE 2011 kritisch zu lesen und in den wissenschaftlichen Dialog einzutreten.

Ihr

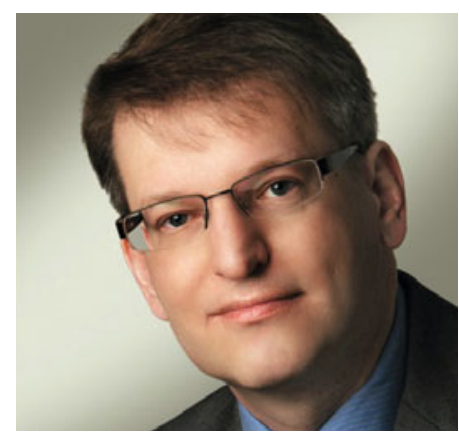

Prof. Dr. med. habil. Jörg Klewer, Zwickau

Schriftleitung

heilberufescience@springer.com

HeilberufeSCIENCE 2011; 2 (2): 41 DOI 10.1007/s16024-011-0234-1 